\title{
Research Article \\ Sufficient Efficiency Conditions for Vector Ratio Problem on the Second-Order Jet Bundle
}

\author{
Ariana Pitea \\ Faculty of Applied Sciences, University "Politehnica" of Bucharest, Splaiul Independentei 313, \\ Bucharest 060042, Romania \\ Correspondence should be addressed to Ariana Pitea, arianapitea@yahoo.com
}

Received 30 January 2012; Accepted 13 February 2012

Academic Editor: Khalida Inayat Noor

Copyright ( $) 2012$ Ariana Pitea. This is an open access article distributed under the Creative Commons Attribution License, which permits unrestricted use, distribution, and reproduction in any medium, provided the original work is properly cited.

\begin{abstract}
Motivated by its possible applications in mechanics and mechanical engineering, in our previous published work (Pitea and Postolache, 2011), we initiated an optimization theory for the second-order jet bundle. We considered the problem of minimization of vectors of curvilinear functionals (well known as mechanical work), thought as multitime multiobjective variational problems, subject to PDE and/or PDI constraints. Within this framework, we introduced necessary optimality conditions. As natural continuation of these results, the present work introduces a study of sufficient efficiency conditions.
\end{abstract}

\section{Introduction}

As it is known, most of the optimization problems arising in practice have several objectives which have to be optimized simultaneously. These problems, of considerable interest, include various branches of mathematical sciences, engineering design, portfolio selection, game theory, decision problems in management science, web access problems, query optimization in databases, and so forth. Also, such kind of optimization problems arise in wide areas of research for new technology as well. First of all, we have in mind the material sciences where many times optimal estimation of material parameters is required, either nondestructive determination of faults is needed. Next, chemistry which provides a huge class of constrained optimization problems such as the determination of contamination sources given the flow model and the variance of the source. Last, but not least, games theory in the main study is finding optimal wining strategies. For descriptions of the web access problem, the portfolio selection problem, and capital budgeting problem, see [1] by Chinchuluun and Pardalos, [2] by Chinchuluun et al., and some references therein. 
In time, several authors have been interested in the study of (vector) ratio programs in connection with generalized convexity. This study is motivated by many practical optimization problems whose objective functions are quotients of two functions. For advances on single-objective ratio programs, see [3] by Khan and Hanson, and [4] by Reddy and Mukherjee, which utilized invexity assumptions in the sense of Hanson [5] to obtain optimality conditions and duality results. As concerning vector ratio problems, Singh and Hanson [6] applied invex functions to derive duality results, while Jeyakumar and Mond [7] generalized these results to the class of $V$-invex functions. Later, Liang et al. [8] introduced a unified formulation of the generalized convexity to derive optimality conditions and duality results for vector ratio problems.

Despite of these important advances in optimization, our multitime multiobjective problem-required by practical reasons-had not been studied so far. In the problem of our very recent study $[9,10]$, the objective vector function is of curvilinear integral type, the integrand depending both on velocities and accelerations, that is why we have chosen as framework the second-order jet bundle [11] (also, see [12]).

This work extends, generalizes, and further develops our research in [13, 14] from the first-order jet bundle (which considers the movement as function of velocities only) to the second-order jet bundle (where the movement is a function both on velocities and accelerations). Note that passing from the first-order jet bundle to the second-order jet bundle is not a facile task, it requests specific techniques to the latter one (e.g., a new quasiinvexity, an appropriate mathematical framework).

Our study is encouraged by its possible application, especially in mechanical engineering, where curvilinear integral objectives are extensively used due to their physical meaning as mechanical work. The objective functions of our type play an essential role in mathematical modeling of certain processes in relation with robotics, tribology, engines, and so forth.

This paper aims to establish some new results on nonlinear optimization on the second order jet bundle. It is organized as follows. In Section 2 our framework is introduced and the minimization problem is described, while in Section 3 sufficient efficiency conditions for our problem are given. Finally, we conclude the paper and suggest possible further development.

\section{Our Framework and Problem Description}

Let $(T, h)$ and $(M, g)$ be Riemannian manifolds of dimensions $p$ and $n$, respectively. The local coordinates on $T$ and $M$ will be written $t=\left(t^{\alpha}\right)$ and $x=\left(x^{i}\right)$, respectively. Let $J^{2}(T, M)$ be the second-order jet bundle associated to $T$ and $M$, see [11].

Throughout this work, we use the customary relations between two vectors of the same dimension, [9]. With the product-order relation on $\mathbb{R}^{p}$, the hyperparallelepiped $\Omega_{t_{0}, t_{1}}$, in $\mathbb{R}^{p}$, with the diagonal opposite points $t_{0}=\left(t_{0}^{1}, \ldots, t_{0}^{p}\right)$ and $t_{1}=\left(t_{1}^{1}, \ldots, t_{1}^{p}\right)$, can be written as interval $\left[t_{0}, t_{1}\right]$. Suppose $\gamma_{t_{0}, t_{1}}$ is a piecewise $C^{2}$-class curve joining the points $t_{0}$ and $t_{1}$.

\section{Important note}

To simplify the presentation, in our subsequent theory, we shall set

$$
\pi_{x}(t)=\left(t, x(t), x_{\gamma}(t), x_{\theta \sigma}(t)\right), \quad \pi_{x^{\circ}}(t)=\left(t, x^{\circ}(t), x_{\gamma}^{\circ}(t), x_{\theta \sigma}^{\circ}(t)\right)
$$


where $x_{\gamma}(t)=\left(\partial x / \partial t^{\gamma}\right)(t), \gamma=\overline{1, p}$, and $x_{\theta \sigma}(t)=\left(\partial^{2} x / \partial t^{\theta} \partial t^{\sigma}\right)(t), \theta, \sigma=\overline{1, p}$, are partial velocities and partial accelerations respectively.The closed Lagrange 1-form densities of $C^{\infty}$ class:

$$
f_{\alpha}=\left(f_{\alpha}^{\ell}\right): J^{2}(T, M) \longrightarrow \mathbb{R}^{r}, \quad k_{\alpha}=\left(k_{\alpha}^{\ell}\right): J^{2}(T, M) \longrightarrow \mathbb{R}^{r}, \quad \ell=\overline{1, r}, \alpha=\overline{1, p},
$$

determine the following path independent functionals, respectively,

$$
F^{\ell}(x(\cdot))=\int_{\gamma_{t_{0}, t_{1}}} f_{\alpha}^{\ell}\left(\pi_{x}(t)\right) d t^{\alpha}, \quad K^{\ell}(x(\cdot))=\int_{\gamma_{t_{0}, t_{1}}} k_{\alpha}^{\ell}\left(\pi_{x}(t)\right) d t^{\alpha} .
$$

We accept that the Lagrange matrix densities:

$$
g=\left(g_{a}^{\mathrm{b}}\right): J^{2}(T, M) \longrightarrow \mathbb{R}^{\mathrm{md}}, \quad a=\overline{1, d}, \quad b=\overline{1, m}, \quad m<n,
$$

of $C^{\infty}$-class define the partial differential inequations (PDI of evolution)

$$
g\left(\pi_{x}(t)\right) \geqq 0, \quad t \in \Omega_{t_{0}, t_{1}}
$$

and the Lagrange matrix densities

$$
h=\left(h_{a}^{b}\right): J^{2}(T, M) \longrightarrow \mathbb{R}^{q d}, \quad a=\overline{1, d}, \quad b=\overline{1, q}, q<n,
$$

defines the partial differential equations (PDE) (of evolution)

$$
h\left(\pi_{x}(t)\right)=0, \quad t \in \Omega_{t_{0}, t_{1}} .
$$

Let $C^{\infty}\left(\Omega_{t_{0}, t_{1}}, M\right)$ be the space of all functions $x: \Omega_{t_{0}, t_{1}} \longrightarrow M$ of $C^{\infty}$-class, with the norm

$$
\|x\|=\|x\|_{\infty}+\sum_{\alpha=1}^{p}\left\|x_{\alpha}\right\|_{\infty}+\sum_{\theta, \sigma=1}^{p}\left\|x_{\theta \sigma}\right\|_{\infty} .
$$

For each $\ell=\overline{1, r}$, suppose $K^{\ell}(x(\cdot))>0$, and consider

$$
\frac{F(x(\cdot))}{K(x(\cdot))}=\left(\frac{F^{1}(x(\cdot))}{K^{1}(x(\cdot))}, \ldots, \frac{F^{r}(x(\cdot))}{K^{r}(x(\cdot))}\right) .
$$

With conditions (2.5) and (2.7), we denote by

$$
\begin{gathered}
\mathcal{F}\left(\Omega_{t_{0}, t_{1}}\right)=\left\{x \in C^{\infty}\left(\Omega_{t_{0}, t_{1}}, M\right) \mid x\left(t_{0}\right)=x_{0}, x\left(t_{1}\right)=x_{1}, g\left(\pi_{x}(t)\right) \leqq 0,\right. \\
\left.h\left(\pi_{x}(t)\right)=0, t \in \Omega_{t_{0}, t_{1}}\right\}
\end{gathered}
$$


the set of all feasible solutions of problem;

$$
(\mathrm{MFP})\left\{\begin{array}{l}
\min \frac{F(x(\cdot))}{K(x(\cdot))} \\
\text { subject to } x(\cdot) \in \mathcal{F}\left(\Omega_{t_{0}, t_{1}}\right),
\end{array}\right.
$$

a PDI and/or PDE-constrained minimum problem.Using the terminology from analytical mechanics, in MFP there are given numbers of $r$ sources, each of them producing mechanical work. This one have to be minimized on a set of limited resources, namely, $\mathcal{F}\left(\Omega_{t_{0}, t_{1}}\right)$.In our previous work [9], we introduced necessary efficiency conditions for problem (MFP). In Section 3, we would like to further develop these results by introducing sufficient efficiency conditions for problem (MFP).

\section{Main Results}

Definition 3.1. A feasible solution $x^{\circ}(\cdot) \in \mathcal{F}\left(\Omega_{t_{0}, t_{1}}\right)$ is called efficient for program (MFP) if and only if for any feasible solution $x(\cdot) \in \mathcal{F}\left(\Omega_{t_{0}, t_{1}}\right)$ :

$$
\frac{F(x(\cdot))}{K(x(\cdot))} \leqq \frac{F\left(x^{\circ}(\cdot)\right)}{K\left(x^{\circ}(\cdot)\right)} \Longrightarrow \frac{F(x(\cdot))}{K(x(\cdot))}=\frac{F\left(x^{\circ}(\cdot)\right)}{K\left(x^{\circ}(\cdot)\right)}
$$

Consider that $x^{\circ}(\cdot) \in \mathcal{F}\left(\Omega_{t_{0}, t_{1}}\right)$ is an efficient solution of problem (MFP). In a very recent article [9], Pitea and Postolache proved that there exist $\Lambda^{10}, \Lambda^{20} \in \mathbb{R}^{r}$ and the smooth functions $\mu^{\circ}: \Omega_{t_{0}, t_{1}} \rightarrow \mathbb{R}^{m d p}, v^{\circ}: \Omega_{t_{0}, t_{1}} \rightarrow \mathbb{R}^{q d p}$, such that

$$
\begin{aligned}
\left\langle\Lambda^{1 \circ}, \frac{\partial f_{\alpha}}{\partial x}\left(\pi_{x^{\circ}}(t)\right)\right\rangle-\left\langle\Lambda^{2 \circ}, \frac{\partial k_{\alpha}}{\partial x}\left(\pi_{x^{\circ}}(t)\right)\right\rangle+\left\langle\mu_{\alpha}^{\circ}(t), \frac{\partial g}{\partial x}\left(\pi_{x^{\circ}}(t)\right)\right\rangle+\left\langle v_{\alpha}^{\circ}(t), \frac{\partial h}{\partial x}\left(\pi_{x^{\circ}}(t)\right)\right\rangle \\
-D_{\gamma}\left(\left\langle\Lambda^{1 \circ}, \frac{\partial f_{\alpha}}{\partial x_{\gamma}}\left(\pi_{x^{\circ}}(t)\right)\right\rangle-\left\langle\Lambda^{2 \circ}, \frac{\partial k_{\alpha}}{\partial x_{\gamma}}\left(\pi_{x^{\circ}}(t)\right)\right\rangle\right. \\
\left.+\left\langle\mu_{\alpha}^{\circ}\left(\pi_{x^{\circ}}(t)\right), \frac{\partial g}{\partial x_{\gamma}}\left(\pi_{x^{\circ}}(t)\right)\right\rangle+\left\langle v_{\alpha}^{\circ}(t), \frac{\partial h}{\partial x_{\gamma}}\left(\pi_{x^{\circ}}(t)\right)\right\rangle\right) \\
+D_{\theta \sigma}^{2}\left(\left\langle\Lambda^{1 \circ}, \frac{\partial f_{\alpha}}{\partial x_{\theta \sigma}}\left(\pi_{x^{\circ}}(t)\right)\right\rangle-\left\langle\Lambda^{2 \circ}, \frac{\partial k_{\alpha}}{\partial x_{\theta \sigma}}\left(\pi_{x^{\circ}}(t)\right)\right\rangle\right. \\
\left.+\left\langle\mu_{\alpha}^{\circ}\left(\pi_{x^{\circ}}(t)\right), \frac{\partial g}{\partial x_{\theta \sigma}}\left(\pi_{x^{\circ}}(t)\right)\right\rangle+\left\langle v_{\alpha}^{\circ}(t), \frac{\partial h}{\partial x_{\theta \sigma}}\left(\pi_{x^{\circ}}(t)\right)\right\rangle\right)=0, \\
t \in \Omega_{t_{0}, t_{1}, \alpha}=\frac{1, p}{1, p}
\end{aligned}
$$

\section{A Natural Question Arises.}

Are these conditions sufficient for $x^{\circ}(\cdot)$ to be an efficient solution for program (MFP)?

To develop our theory, we have to introduce an appropriate generalized convexity. 
Let $\rho$ be a real number, $b: C^{\infty}\left(\Omega_{t_{0}, t_{1}}, M\right) \times C^{\infty}\left(\Omega_{t_{0}, t_{1}}, M\right) \rightarrow[0, \infty)$ a functional, and $a=\left(a_{\alpha}\right), \alpha=\overline{1, p}$, a closed 1 -form. To $a$, we associate the curvilinear integral.

$$
A(x(\cdot))=\int_{\gamma_{t_{0}, t_{1}}} a_{\alpha}\left(\pi_{x}(t)\right) d t^{\alpha}
$$

Definition 3.2. The functional $A$ is called [strictly] $(\rho, b)$-quasiinvex at the point $x^{\circ}(\cdot)$ if there exists a vector function $\eta: J^{2}\left(\Omega_{t_{0}, t_{1}}, M\right) \times J^{2}\left(\Omega_{t_{0}, t_{1}}, M\right) \rightarrow \mathbb{R}^{n}$, vanishing at the point $\left(\pi_{x^{\circ}}(t), \pi_{x^{\circ}}(t)\right)$, and the function $\theta$ defined on $C^{\infty}\left(\Omega_{t_{0}, t_{1}}, M\right) \times C^{\infty}\left(\Omega_{t_{0}, t_{1}}, M\right)$ to $\mathbb{R}^{n}$, such that for any $x(\cdot)\left[x(\cdot) \neq x^{\circ}(\cdot)\right]$, the following implication holds:

$$
\begin{aligned}
&\left(A(x(\cdot)) \leq A\left(x^{\circ}(\cdot)\right)\right) \Longrightarrow \\
&\left(b x\left((\cdot), x^{\circ}(\cdot)\right) \int_{\gamma_{t_{0}, t_{1}}}[\right.\left\langle\left(\pi_{x}(t), \pi_{x^{\circ}}(t)\right), \frac{\partial a_{\alpha}}{\partial x}\left(\pi_{x^{\circ}}(t)\right)\right\rangle \\
&+\left\langle D_{\gamma} \eta\left(\pi_{x}(t), \pi_{x^{\circ}}(t)\right), \frac{\partial a_{\alpha}}{\partial x_{\gamma}}\left(\pi_{x^{\circ}}(t)\right)\right\rangle \\
&\left.+\left\langle D_{\theta \sigma}^{2} \eta\left(\pi_{x}(t), \pi_{x^{\circ}}(t)\right), \frac{\partial a_{\alpha}}{\partial x_{\theta \sigma}}\left(\pi_{x^{\circ}}(t)\right)\right\rangle\right] d t^{\alpha} \\
& {\left.[<] \leq-\rho b\left(x(\cdot), x^{\circ}(\cdot)\right)\left\|\theta\left(x(\cdot), x^{\circ}(\cdot)\right)\right\|^{2}\right) . }
\end{aligned}
$$

The quasiinvexity is used, in appropriate forms, in recent works for studies of some multiobjective programming problems, see [15-17] by Mititelu et al., [18] by Nahak and Mohapatra.

Now, we can establish efficiency sufficient conditions for problem (MFP).

Theorem 3.3. Consider the vectors $\Lambda^{10}, \Lambda^{20}$ from $\mathbb{R}^{r}$ and the functions $x^{\circ}(\cdot), \mu^{\circ}(\cdot), v^{\circ}(\cdot)$ satisfying conditions (3.2). Suppose that the following properties hold:

(a) the functional $\left\langle\Lambda^{10}, F(x(\cdot))\right\rangle-\left\langle\Lambda^{2 \circ}, K(x(\cdot))\right\rangle$ is $\left(\rho_{1}, b\right)$-quasiinvex at the point $x^{\circ}(\cdot)$ with respect to $\eta$ and $\theta$;

(b) the functional $\int_{\gamma_{0, t_{1}}}\left\langle\mu_{\alpha}^{\circ}(t), g\left(\pi_{x}(t)\right)\right\rangle d t^{\alpha}$ is $\left(\rho_{2}, b\right)$-quasiinvex at the point $x^{\circ}(\cdot)$ with respect to $\eta$ and $\theta$;

(c) the functional $\int_{\gamma_{t_{0}, t_{1}}}\left\langle v_{\alpha}^{\circ}(t), h\left(\pi_{x}(t)\right)\right\rangle d t^{\alpha}$ is $\left(\rho_{3}, b\right)$-quasiinvex at the point $x^{\circ}(\cdot)$ with respect to $\eta$ and $\theta$;

(d) one of the integrals of $(a)$ - (c) is strictly $\left(\rho_{1}, b\right),\left(\rho_{2}, b\right)$ or $\left(\rho_{3}, b\right)$-quasiinvex at the point $x^{\circ}(\cdot)$ with respect to $\eta$ and $\theta$;

(e) $\rho_{1}+\rho_{2}+\rho_{3} \geq 0$;

(f) $\Lambda_{\ell}^{1 \circ} F^{\ell}\left(x^{\circ}(\cdot)\right)-\Lambda_{\ell}^{2 \circ} K^{\ell}\left(x^{\circ}(\cdot)\right)=0$, for each $\ell=\overline{1, r}$.

Then the point $x^{\circ}(\cdot)$ is an efficient solution of problem (MFP). 
Proof. Let us suppose that the point $x^{\circ}(\cdot)$ is not an efficient solution for problem (MFP). Then, there is a feasible solution $x(\cdot)$ for problem (MFP), such that

$$
\frac{F^{\ell}(x(\cdot))}{K^{\ell}(x(\cdot))} \leq \frac{F^{\ell}\left(x^{\circ}(\cdot)\right)}{K^{\ell}\left(x^{\circ}(\cdot)\right)}, \quad \ell=\overline{1, r}
$$

the case $x(\cdot)=x^{\circ}(\cdot)$ being excluded. That is:

$$
\Lambda_{\ell}^{1 \circ} F^{\ell}(x(\cdot))-\Lambda_{\ell}^{2 \circ} K^{\ell}(x(\cdot)) \leq \Lambda_{\ell}^{1 \circ} F^{\ell}\left(x^{\circ}(\cdot)\right)-\Lambda_{\ell}^{2 \circ} K^{\ell}\left(x^{\circ}(\cdot)\right), \quad \ell=\overline{1, r} .
$$

Making the sum after $\ell=\overline{1, r}$, we get

$$
\left\langle\Lambda^{1 \circ}, F(x(\cdot))\right\rangle-\left\langle\Lambda^{2 \circ}, K(x(\cdot))\right\rangle \leq\left\langle\Lambda^{1 \circ}, F\left(x^{\circ}(\cdot)\right)\right\rangle-\left\langle\Lambda^{2 \circ}, K\left(x^{\circ}(\cdot)\right)\right\rangle .
$$

According to condition (a), it follows that

$$
\begin{aligned}
b\left(x(\cdot), x^{\circ}(\cdot)\right) \int_{\gamma_{t_{0}, t_{1}}}\left[\left\langle\eta\left(\pi_{x}(t), \pi_{x^{\circ}}(t)\right),\left\langle\Lambda^{1 \circ}, \frac{\partial f_{\alpha}}{\partial x}\left(\pi_{x^{\circ}}(t)\right)\right\rangle-\left\langle\Lambda^{2 \circ}, \frac{\partial k_{\alpha}}{\partial x}\left(\pi_{x^{\circ}}(t)\right)\right\rangle\right\rangle\right. \\
+\left\langle D_{\gamma} \eta\left(\pi_{x}(t), \pi_{x^{\circ}}(t)\right),\left\langle\Lambda^{1 \circ}, \frac{\partial f_{\alpha}}{\partial x_{\gamma}}\left(\pi_{x}(t)\right)\right\rangle-\left\langle\Lambda^{2 \circ}, \frac{\partial k_{\alpha}}{\partial x_{\gamma}}\left(\pi_{x^{\circ}}(t)\right)\right\rangle\right\rangle \\
\left.+\left\langle D_{\theta \sigma}^{2} \eta\left(\pi_{x}(t), \pi_{x^{\circ}}(t)\right),\left\langle\Lambda^{1 \circ}, \frac{\partial f_{\alpha}}{\partial x_{\theta \sigma}}\left(\pi_{x}(t)\right)\right\rangle-\left\langle\Lambda^{2 \circ}, \frac{\partial k_{\alpha}}{\partial x_{\theta \sigma}}\left(\pi_{x^{\circ}}(t)\right)\right\rangle\right\rangle\right] d t^{\alpha} \\
\leqq-\rho_{1} b\left(x(\cdot), x^{\circ}(\cdot)\right)\left\|\theta\left(x(\cdot), x^{\circ}(\cdot)\right)\right\|^{2} .
\end{aligned}
$$

Applying property (b), the inequality

$$
\int_{\gamma_{t_{0}, t_{1}}}\left\langle\mu_{\alpha}^{\circ}(t), g\left(\pi_{x}(t)\right)\right\rangle d t^{\alpha} \leqq \int_{\gamma_{t_{0}, t_{1}}}\left\langle\mu_{\alpha}^{\circ}(t), g\left(\pi_{x^{\circ}}(t)\right)\right\rangle d t^{\alpha}
$$

leads us to

$$
\begin{aligned}
& b\left(x(\cdot), x^{\circ}(\cdot)\right) \int_{\gamma_{t_{0}, t_{1}}}(\langle\left.\eta\left(\pi_{x}(t), \pi_{x^{\circ}}(t)\right),\left\langle\mu_{\alpha}^{\circ}(t), \frac{\partial g}{\partial x}\left(\pi_{x^{\circ}}(t)\right)\right\rangle\right\rangle \\
&+\left\langle D_{\gamma} \eta\left(\pi_{x}(t), \pi_{x^{\circ}}(t)\right),\left\langle\mu_{\alpha}^{\circ}(t), \frac{\partial g}{\partial x_{\gamma}}\left(\pi_{x^{\circ}}(t)\right)\right\rangle\right\rangle \\
&+\left.\left\langle D_{\theta \sigma}^{2} \eta\left(\pi_{x}(t), \pi_{x^{\circ}}(t)\right),\left\langle\mu_{\alpha}^{\circ}(t), \frac{\partial g}{\partial x_{\theta \sigma}}\left(\pi_{x^{\circ}}(t)\right)\right\rangle\right\rangle\right) d t^{\alpha} \\
& \leqq-\rho_{2} b\left(x(\cdot), x^{\circ}(\cdot)\right)\left\|\theta\left(x(\cdot), x^{\circ}(\cdot)\right)\right\|^{2} .
\end{aligned}
$$


Taking into account condition (c), the equality

$$
\int_{\gamma_{t_{0}, t_{1}}}\left\langle v_{\alpha}^{\circ}(t), h\left(\pi_{x}(t)\right)\right\rangle d t^{\alpha}=\int_{\gamma_{t_{0}, t_{1}}}\left\langle v_{\alpha}^{\circ}(t), h\left(\pi_{x^{\circ}}(t)\right)\right\rangle d t^{\alpha}
$$

implies

$$
\begin{aligned}
& b\left(x(\cdot), x^{\circ}(\cdot)\right) \int_{\gamma_{t_{0}, t_{1}}}(\left\langle\eta\left(\pi_{x}(t), \pi_{x^{\circ}}(t)\right),\left\langle\nu_{\alpha}^{\circ}(t), \frac{\partial h}{\partial x}\left(\pi_{x^{\circ}}(t)\right)\right\rangle\right\rangle \\
&+\left\langle D_{\gamma} \eta\left(\pi_{x}(t), \pi_{x^{\circ}}(t)\right),\left\langle v_{\alpha}^{\circ}(t), \frac{\partial h}{\partial x_{\gamma}}\left(\pi_{x^{\circ}}(t)\right)\right\rangle\right\rangle \\
&\left.+\left\langle D_{\theta \sigma}^{2} \eta\left(\pi_{x}(t), \pi_{x^{\circ}}(t)\right),\left\langle\nu_{\alpha}^{\circ}(t), \frac{\partial h}{\partial x_{\theta \sigma}}\left(\pi_{x^{\circ}}(t)\right)\right\rangle\right\rangle\right) d t^{\alpha} \\
& \leqq-\rho_{3} b\left(x(\cdot), x^{\circ}(\cdot)\right)\left\|\theta\left(x(\cdot), x^{\circ}(\cdot)\right)\right\|^{2} .
\end{aligned}
$$

Summing side by side relations (3.8), (3.10), (3.12) and using condition (d), it follows that

$$
\begin{gathered}
b\left(x(\cdot), x^{\circ}(\cdot)\right) \int_{\gamma_{t_{0}, t_{1}}}\left\langle\eta\left(\pi_{x}(t), \pi_{x^{\circ}}(t)\right),\left\langle\Lambda^{1 \circ}, \frac{\partial f_{\alpha}}{\partial x}\left(\pi_{x^{\circ}}(t)\right)\right\rangle\right. \\
\left.-\left\langle\Lambda^{2 \circ}, \frac{\partial k_{\alpha}}{\partial x}\left(\pi_{x^{\circ}}(t)\right)\right\rangle+\left\langle\mu_{\alpha}^{\circ}(t), \frac{\partial g}{\partial x}\left(\pi_{x^{\circ}}(t)\right)\right\rangle+\left\langle v_{\alpha}^{\circ}(t), \frac{\partial h}{\partial x}\left(\pi_{x^{\circ}}(t)\right)\right\rangle\right\rangle d t^{\alpha} \\
+b\left(x(\cdot), x^{\circ}(\cdot)\right) \int_{\gamma_{t_{0}, t_{1}}}\left\langle D_{\gamma} \eta\left(\pi_{x}(t), \pi_{x^{\circ}}(t)\right),\left\langle\Lambda^{1 \circ}, \frac{\partial f_{\alpha}}{\partial x_{\gamma}}\left(\pi_{x^{\circ}}(t)\right)\right\rangle-\left\langle\Lambda^{2 \circ}, \frac{\partial k_{\alpha}}{\partial x_{\gamma}}\left(\pi_{x^{\circ}}(t)\right)\right\rangle\right. \\
\left.+\left\langle\mu_{\alpha}^{\circ}(t), \frac{\partial g}{\partial x_{\gamma}}\left(\pi_{x^{\circ}}(t)\right)\right\rangle\right\rangle d t^{\alpha}+\left\langle v_{\alpha}^{\circ}(t), \frac{\partial h}{\partial x_{\gamma}}\left(\pi_{x^{\circ}}(t)\right)\right\rangle \\
+b\left(x(\cdot), x^{\circ}(\cdot)\right) \int_{\gamma_{t_{0}, t_{1}}}\left\langle D_{\theta \sigma}^{2} \eta\left(\pi_{x}(t), \pi_{x^{\circ}}(t)\right),\left\langle\Lambda^{1 \circ}, \frac{\partial f_{\alpha}}{\partial x_{\theta \sigma}}\left(\pi_{x^{\circ}}(t)\right)\right\rangle-\left\langle\Lambda^{2 \circ}, \frac{\partial k_{\alpha}}{\partial x_{\theta \sigma}}\left(\pi_{x^{\circ}}(t)\right)\right\rangle\right. \\
\left.+\left\langle\mu_{\alpha}^{\circ}(t), \frac{\partial g}{\partial x_{\theta \sigma}}\left(\pi_{x^{\circ}}(t)\right)\right\rangle\right\rangle d t^{\alpha}+\left\langle v_{\alpha}^{\circ}(t), \frac{\partial h}{\partial x_{\theta \sigma}}\left(\pi_{x^{\circ}}(t)\right)\right\rangle \\
<-\left(\rho_{1}+\rho_{2}+\rho_{3}\right) b\left(x(\cdot), x^{\circ}(\cdot)\right)\left\|\theta\left(x(\cdot), x^{\circ}(\cdot)\right)\right\|^{2} .
\end{gathered}
$$

This inequality implies that $\left.b\left(x(\cdot), x^{\circ}(\cdot)\right)\right\rangle 0$, therefore, we obtain

$$
\begin{aligned}
\int_{\gamma_{t_{0}, t_{1}}}\left\langle\eta\left(\pi_{x}(t), \pi_{x^{\circ}}(t)\right)\left\langle\Lambda^{1 \circ}, \frac{\partial f_{\alpha}}{\partial x}\left(\pi_{x^{\circ}}(t)\right)\right\rangle-\left\langle\Lambda^{2 \circ}, \frac{\partial k_{\alpha}}{\partial x}\left(\pi_{x^{\circ}}(t)\right)\right\rangle\right. \\
\left.+\left\langle\mu_{\alpha}^{\circ}(t), \frac{\partial g}{\partial x}\left(\pi_{x^{\circ}}(t)\right)\right\rangle+\left\langle v_{\alpha}^{\circ}(t), \frac{\partial h}{\partial x}\left(\pi_{x^{\circ}}(t)\right)\right\rangle\right\rangle d t^{\alpha} \\
+\int_{\gamma_{t_{0}, t_{1}}}\left\langle D_{\gamma} \eta\left(\pi_{x}(t), \pi_{x^{\circ}}(t)\right)\left\langle\Lambda^{1 \circ}, \frac{\partial f_{\alpha}}{\partial x_{\gamma}}\left(\pi_{x^{\circ}}(t)\right)\right\rangle-\left\langle\Lambda^{2 \circ}, \frac{\partial k_{\alpha}}{\partial x_{\gamma}}\left(\pi_{x^{\circ}}(t)\right)\right\rangle\right.
\end{aligned}
$$




$$
\begin{gathered}
\left.+\left\langle\mu_{\alpha}^{\circ}(t), \frac{\partial g}{\partial x_{\gamma}}\left(\pi_{x^{\circ}}(t)\right)\right\rangle+\left\langle v_{\alpha}^{\circ}(t), \frac{\partial h}{\partial x_{\gamma}}\left(\pi_{x^{\circ}}(t)\right)\right\rangle\right\rangle d t^{\alpha} \\
+\int_{\gamma_{t_{0}, t_{1}}}\left\langle D_{\theta \sigma}^{2} \eta\left(\pi_{x}(t), \pi_{x^{\circ}}(t)\right),\left\langle\Lambda^{1 \circ}, \frac{\partial f_{\alpha}}{\partial x_{\theta \sigma}}\left(\pi_{x^{\circ}}(t)\right)\right\rangle-\left\langle\Lambda^{2 \circ}, \frac{\partial k_{\alpha}}{\partial x_{\theta \sigma}}\left(\pi_{x^{\circ}}(t)\right)\right\rangle\right. \\
\left.+\left\langle\mu_{\alpha}^{\circ}(t), \frac{\partial g}{\partial x_{\theta \sigma}}\left(\pi_{x^{\circ}}(t)\right)\right\rangle+\left\langle v_{\alpha}^{\circ}(t), \frac{\partial h}{\partial x_{\theta \sigma}}\left(\pi_{x^{\circ}}(t)\right)\right\rangle\right\rangle d t^{\alpha} \\
<-\left(\rho_{1}+\rho_{2}+\rho_{3}\right)\left\|\theta\left(x(\cdot), x^{\circ}(\cdot)\right)\right\|^{2} .
\end{gathered}
$$

According to [19], we have the following.

Lemma 3.4. A total divergence is equal to a total derivative.

After integrating by parts the last two integrals, the left-hand side of the previous inequality can be written as the sum of two integrals. The former has as integrant the scalar product between $\eta\left(\pi_{x}(t), \pi_{x^{\circ}}(t)\right)$ and the null expression from (3.2). The later is also null, being and integral from a total derivative by Lemma 3.4. Therefore, the previous inequality leads us to a contradiction, that is $0<-\left(\rho_{1}+\rho_{2}+\rho_{3}\right)\left\|\theta\left(x(\cdot), x^{\circ}(\cdot)\right)\right\|^{2}$. Thus, the point $x^{\circ}(\cdot)$ is an efficient solution for problem (MFP).

Replacing the integrals from hypotheses (b) and (c), of Theorem 3.3, by the integral

$$
\int_{\gamma_{t_{0}, t_{1}}}\left[\left\langle\mu_{\alpha}^{\circ}(t), g\left(\pi_{x}(t)\right)\right\rangle+\left\langle v_{\alpha}^{\circ}(t), h\left(\pi_{x}(t)\right)\right\rangle\right] d t^{\alpha},
$$

the following statement is obtained.

Corollary 3.5. Let $x^{\circ}(\cdot)$ be a feasible solution of problem $(M F P), \mu^{\circ}(\cdot), \nu^{\circ}(\cdot)$ be functions, and $\Lambda^{1 \circ}$, $\Lambda^{20}$ vectors from $\mathbb{R}^{r}$ such that relations (3.2) are satisfied. Suppose that the following conditions are fulfilled:

(a) the functional $\left\langle\Lambda^{10}, F(x(\cdot))\right\rangle-\left\langle\Lambda^{2 \circ}, K(x(\cdot))\right\rangle$ is $\left(\rho_{1}, b\right)$-quasiinvex at the point $x^{\circ}(\cdot)$ with respect to $\eta$ and $\theta$;

(b) the functional $\int_{\gamma_{t_{0}, t_{1}}}\left[\left\langle\mu_{\alpha}^{\circ}(t), g\left(\pi_{x}(t)\right)\right\rangle+\left\langle v_{\alpha}^{\circ}(t), h\left(\pi_{x}(t)\right)\right\rangle\right] d t^{\alpha}$ is $\left(\rho_{2}, b\right)$-quasiinvex at the point $x^{\circ}(\cdot)$ with respect to $\eta$ and $\theta$;

(c) one of the integrals from (a) or (b) is strictlyquasiinvex at the point $x^{\circ}(\cdot)$;

(d) $\rho_{1}+\rho_{2} \geq 0$;

(e) $\Lambda_{\ell}^{1 \circ} F^{\ell}\left(x^{\circ}(\cdot)\right)-\Lambda_{\ell}^{2 \circ} K^{\ell}\left(x^{\circ}(\cdot)\right)=0$, for each $\ell=\overline{1, r}$.

Then, the point $x^{\circ}(\cdot)$ is an efficient solution of problem (MFP).

\section{Conclusion and Further Development}

In our work [9], we initiated an optimization theory for the second-order jet bundle. We considered the problem of minimization of vectors of curvilinear functionals (well known 
as mechanical work), thought as multitime multiobjective variational problem, subject to PDE and/or PDI constraints (limited resources). Within this framework, we introduced necessary conditions. As natural continuation of our results in [9], and strongly motivated by its possible applications in mechanics, the present work introduced a study of sufficient efficiency conditions for (MFP).

Since ratio programming problems with objective function of our type arise from applied areas as decision problems in management, game theory, engineering studies, and design, we will orient our future research to the development of (strong) dual program theory for these problems [20].

\section{References}

[1] A. Chinchuluun and P. M. Pardalos, "A survey of recent developments in multiobjective optimization," Annals of Operations Research, vol. 154, pp. 29-50, 2007.

[2] A. Chinchuluun, P. M. Pardalos, A. Migdalas, and L. Pitsoulis, Pareto Optimality, Game Theory and Equilibria, vol. 17 of Springer Optimization and Its Applications, Springer, New York, NY, USA, 2008, Edited by Altannar Chinchuluun, Panos M. Pardalos, Athanasios Migdalas and Leonidas Pitsoulis.

[3] Z. A. Khan and M. A. Hanson, "On ratio invexity in mathematical programming," Journal of Mathematical Analysis and Applications, vol. 205, no. 2, pp. 330-336, 1997.

[4] L. V. Reddy and R. N. Mukherjee, "Some results on mathematical programming with generalized ratio invexity," Journal of Mathematical Analysis and Applications, vol. 240, no. 2, pp. 299-310, 1999.

[5] M. A. Hanson, "A duality theorem in non-linear programming with non-linear constraints," The Australian Journal of Statistics, vol. 3, pp. 64-72, 1961.

[6] C. Singh and M. A. Hanson, "Multiobjective fractional programming duality theory," Naval Research Logistics, vol. 38, no. 6, pp. 925-933, 1991.

[7] V. Jeyakumar and B. Mond, "On generalised convex mathematical programming," Australian Mathematical Society Journal Series B, vol. 34, no. 1, pp. 43-53, 1992.

[8] Z.-A. Liang, H.-X. Huang, and P. M. Pardalos, "Efficiency conditions and duality for a class of multiobjective fractional programming problems," Journal of Global Optimization, vol. 27, no. 4, pp. 447-471, 2003.

[9] A. Pitea and M. Postolache, "Minimization of vectors of curvilinear functionals on the second order jet bundle. Necessary conditions," Optimization Letters, vol. 6, no. 3, pp. 459-470, 2011.

[10] A. Pitea, Integral geometry and PDE constrained optimization problems, Ph.D. thesis, University "Politehnica" of Bucharest, 2008.

[11] C. Udrişte and M. Postolache, Atlas of Magnetic Geometric Dynamics, vol. 3, Geometry Balkan Press, Bucharest, Romania, 2001.

[12] C. Udrişte, O. Dogaru, and I. Ţ Tevy, "Null Lagrangian forms and Euler-Lagrange PDEs," Journal of Advanced Mathematical Studies, vol. 1, no. 1-2, pp. 143-156, 2008.

[13] A. Pitea and M. Postolache, "Duality theorems for a new class of multitime multiobjective variational problems," Journal of Global Optimization. In press.

[14] A. Pitea and C. Udrişte, "Sufficient efficiency conditions for a minimizing fractional program," "Politehnica" University of Bucharest. Scientific Bulletin, vol. 72, no. 2, pp. 13-20, 2010.

[15] S.. Mititelu, "Extensions in invexity theory," Journal of Advanced Mathematical Studies, vol. 1, no. 1-2, pp. 63-70, 2008.

[16] Ş. Mititelu and M. Postolache, "Mond-Weir dualities with Lagrangians for multiobjective fractional and non-fractional variational problems," Journal of Advanced Mathematical Studies, vol. 3, no. 1, pp. 41-58, 2010.

[17] Ş. Mititelu, V. Preda, and M. Postolache, "Duality of multitime vector integral programming with quasiinvexity," Journal of Advanced Mathematical Studies, vol. 4, no. 2, pp. 59-72, 2011.

[18] C. Nahak and R. N. Mohapatra, "Nonsmooth $\rho-(\eta, \theta)$-invexity in multiobjective programming problems," Optimization Letters, vol. 6, no. 2, pp. 253-260, 2012.

[19] A. Pitea, "Null Lagrangian forms on 2nd order jet bundles," Journal of Advanced Mathematical Studies, vol. 3, no. 1, pp. 73-82, 2010.

[20] M. Postolache, "Minimization of vectors of curvilinear functionals on second order jet bundle: dual program theory," Abstract and Applied Analysis, vol. 2012, Article ID 535416, 12 pages, 2012. 


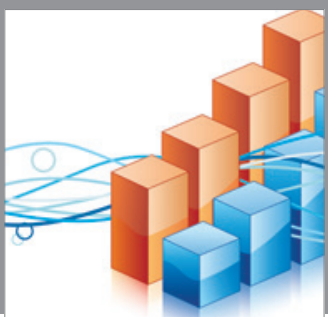

Advances in

Operations Research

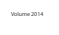

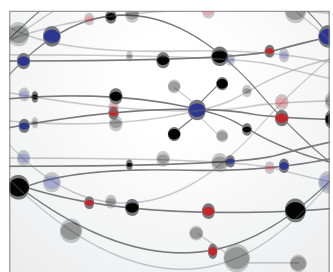

\section{The Scientific} World Journal
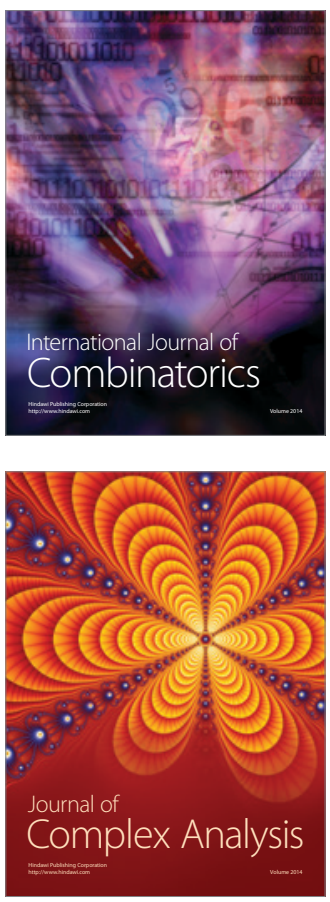

International Journal of

Mathematics and

Mathematical

Sciences
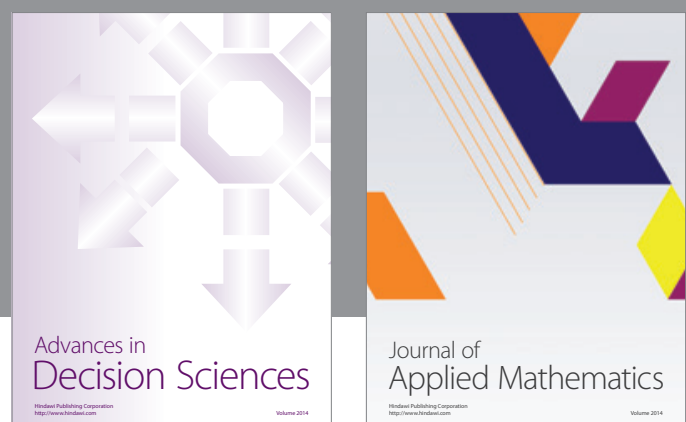

Journal of

Applied Mathematics
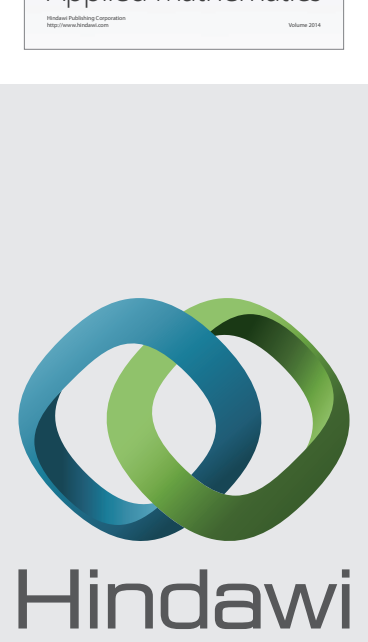

Submit your manuscripts at http://www.hindawi.com
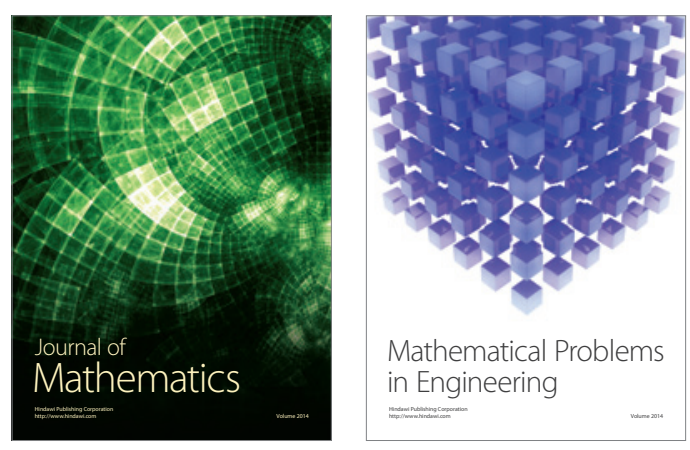

Mathematical Problems in Engineering
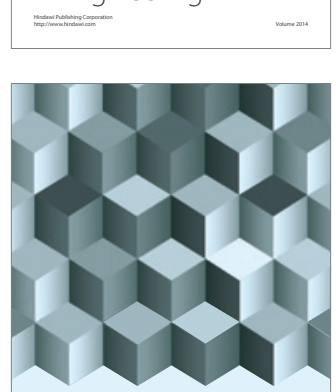

Journal of

Function Spaces
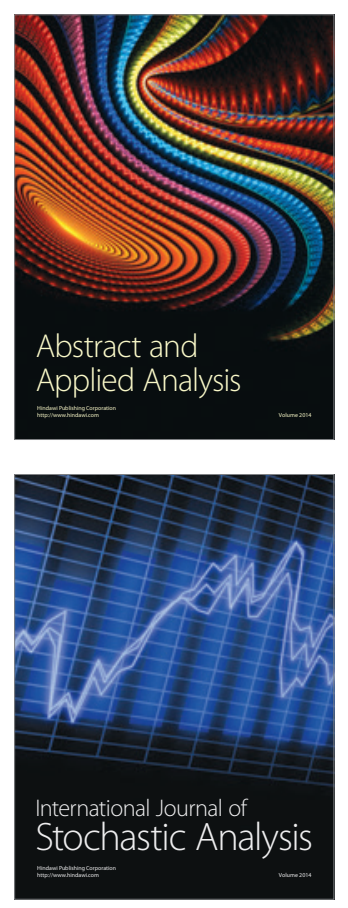

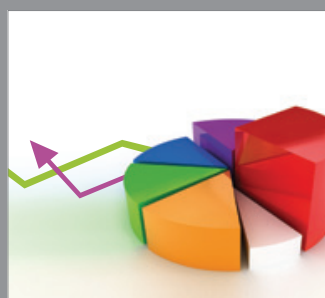

ournal of

Probability and Statistics

Promensencen
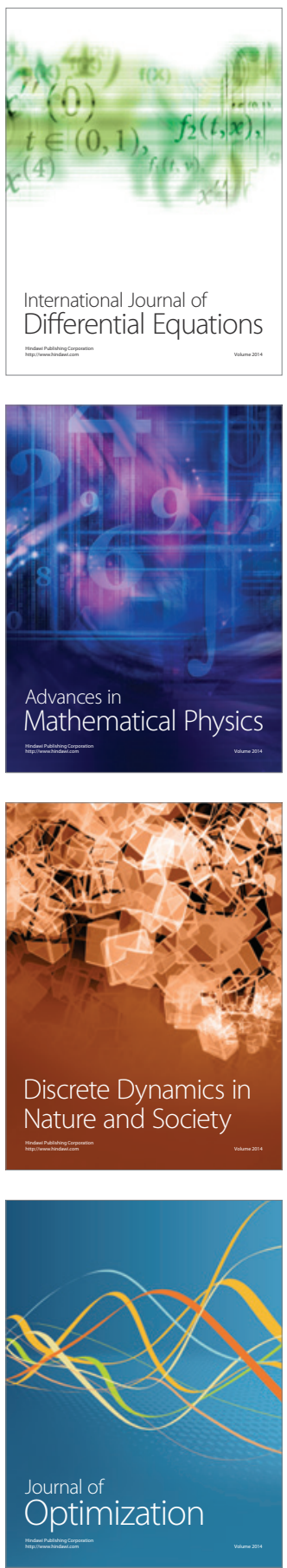